\title{
Controlling Unmanned Surface Vehicle Using MQTT Protocol
}

\author{
Rachmad Andri Atmoko ${ }^{1,2}$, Daoguo Yang ${ }^{1}$, M Yayan Alfiani ${ }^{3}$, Lilik Subiyanto ${ }^{3}$ \\ ${ }^{1}$ School of Mechanical and Electrical Engineering, Guilin University of Electronic \\ Technology, Guilin, China \\ ${ }^{2}$ Faculty of Engineering, University of Jember, Jember, Indonesia \\ ${ }^{3}$ Automation Engineering Department, Shipbuilding Institute of Polytechnic Surabaya, \\ Surabaya, Indonesia
}

\section{E-mail: mokoraden@hotmail.com}

\begin{abstract}
The communication system at USV is the most important part of ensuring that the USV control system runs well. Communication systems that are commonly used at USV are generally still local networks. The development of a wider range of communications is needed to improve USV scalability so that the benefits can be increased to support the concept of remote laboratory and remote surveillance. USV is generally controlled using a wireless network with a limited work area using WIFI or using another electromagnetic waves transmission. This research proposes the development control and monitoring systems of USV to be controlled over the Internet by using the message queuing telemetry transport(MQTT) Protocol. This idea makes the USV can be controlled remotely, no longer have to operate it in the field. The control speed response of rudder and motor are analyzed. Performance monitoring of the compass sensor, GPS, and cameras also showed good results. As a result, the average delay time generated is 1.6 second.
\end{abstract}

Keywords: unmanned surface vehicle (USV); remote; message queuing telemetry transport (MQTT); internet of things

\section{Introduction}

USV will be one of the critical technologies in the future. There are a variety of benefits obtained by implementing this technology for the military, watershed surveillance, environmental monitoring, and to assist in the field of marine research [1][2]. USV offers advantages to reduce operating costs compared to using conventional boats [3]. USV has helped resolve various problems including for jellyfish removal robot system which can remove jellyfish using an autonomous navigation system [4], USV for antisubmarine warfare [5] and water quality monitoring and measurement [6]. Mostly, USV were used to observe areas of water use local communications such as Zigbee protocol technology that has ultra-low power consumption and low cost [7], and use wireless LAN based on IP addressing [8]. In research [9] uses Wi-Fi and specifically evaluates variations in Wi-Fi link quality at $2.4 \mathrm{GHz}$ and $5.8 \mathrm{GHz}$ between a USV and a BS located onshore. Development of USV communication using Wi-Fi and WiMAX technology has been suggested in the Swarms of Aquatic surfaces drone [10].

The development of the USV control system in the future should not only be operated in the range of local distances but also must be controlled through the internet network. For instance, a USV for patrol of territorial waters can be controlledby users at certain headquarters or branch offices. All that is possible if USV supports the internet of things communication protocol. Research on the development of USV still revolves around control systems. Some of them make the collision avoidance systems design in the sea environment for USV navigation which is adjusted to the COLREGs rule [11], development of PID control system optimization using Particle swarm optimization (PSO) to control USV yaw position (heading) of the model [12], and development autopilot system for USV [13].

The above research has been able to meet real-time data transfer needs at USV. However, its nature is still local communication. This study proposes the use of the MQTT protocol which is widely used in remote control and monitoring applications using the internet network. One of the benefits obtained is that it can make USV support the remote laboratory concept. Nowadays, remote laboratories appear with two main objectives, which is to produce many students to experiment with remote resources and reduce the cost of classroom and labs. Making it easier for the institution to share remote labs [14]. 
Remote labs can also improve distance-learning, improve safety, and improve accessibility. Moreover, this system reduces the time set up, faster results and minimization of distractions. It can be used as a supplement approach for student learning processes [15-17]. This research can help researchers and students in the field of environment who focus on water problems to make observations and retrieve data remotely. Institutions that do not have equipment can also use it with the remote labs share concept. The USV remote can also be used to assist the military and security sectors to carry out security patrols and surveillance which can be done remotely from the head office.

\section{Materials and Methods}

The main objective of this paper is to develop USV control system using MQTT Protocol. The system built is divided into three layers, namely Hardware Layer, Server Layer, and Application Layer.

\subsection{Hardware Layer}

Figure 1 describes the hardware design consisting of sensors, controllers and gateways. We use two different controllers to optimize computing load. Arduino Uno handles interactions with brushless motors and servo motor.

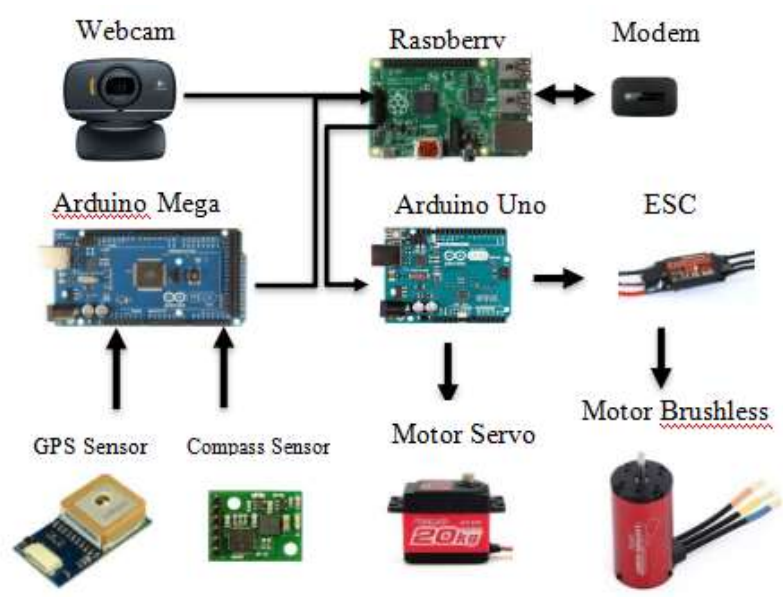

Figure 1. USV Hardware.

Arduino Mega handles communications with GPS and compass sensor. Raspberry mini PC as a gateway that communicates directly to MQTT Brokers. The gateway also handles interactions with web camera and modems. Power supply sources come from Lipo battery and power banks. Web RTC server installed on the gateway. We use easyRTC as WebRTC Server. It is a framework built on the WebRTC protocol which is a standard for real-time communication of audio, data, and video between web browsers. WebRTC uses peer-to-peer communication to capture, transmit, receive, and play audio/video data. In this case, we use it for webcam video data transmission. The USV has a catamaran type. It has two-hull ship design as seen in Figure 2 the following :

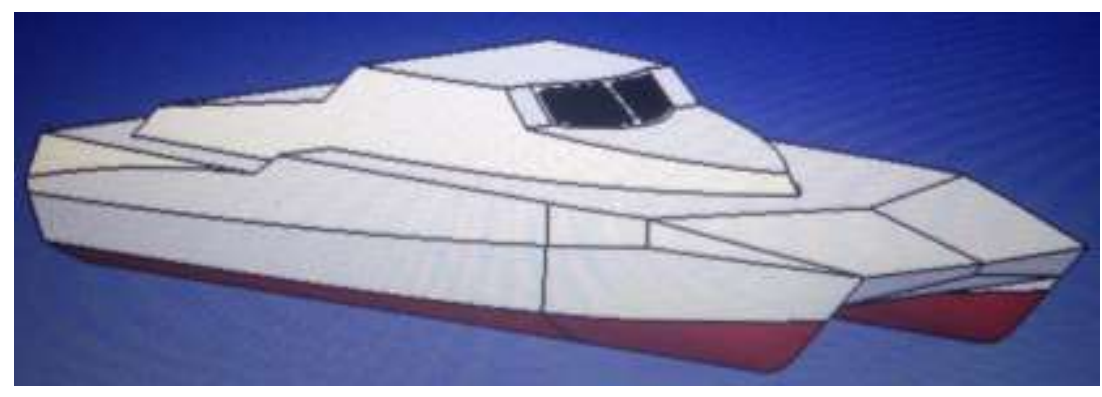

Figure 2. USV Catamaran Design. 
The Arduino Uno contains a program used to control the actuator. The program will read serially transmitted data from the gateway using Python. The data sent includes an order to move the brushless motor actuator and servo motor. The servo motor functions to move the webcam camera to the angle that the user wants. Arduino Mega contains a program that is used to process data obtained from the sensor and then sent to the MQTT broker through the gateway.

\subsection{Server Layer}

At this layer, we use the MQTT Broker Mosquito. The server uses a virtual private server (VPS) with technical specifications in table 1.

TABLE 1

TECHNICAL SPECIFICATION OF SERVER

\begin{tabular}{ll} 
Item & Value \\
\hline CPU Cores & 1 \\
Total CPU Speed & $2.4 \mathrm{Ghz}$ \\
Memory & $1 \mathrm{~Gb}$ \\
Disk Space & $20 \mathrm{~Gb}$ \\
Bandwidth & $1000 \mathrm{~Gb}$ \\
Server Location & Singapore
\end{tabular}

\subsection{Application Layer}

MQTT protocol has a small packet data size compared to other protocols such as HTTP [18-20]. It is suitable for real-time applications. MQTT working with publish/subscribe concept. MQTT broker is required to establish communication between users called subscribers and publishers. Users communicate with each other by publish data to a topic prepared on the MQTT broker. Users who act as subscribers will retrieve data on the specified topic. In this case, user and USV act as publisher and subscriber. Users publish control commands via topic/rudder navigation and /motorspeed. MQTT Brokers receive messages and forward messages to USV who have subscribed to the topic /speedmotor. The message received by USV is the parameter used to control the motor speed and direction of the rudder ordered by the user. USV also acts as a publisher that sends sensor data to topics /gpssensors and /compassensors. MQTT Broker forwards the data to users who have subscribed to the same topic.

In this layer there is a web interface application created using HTML and Javascript programming languages. The appearance of the interface can be seen in the following figure 3 .

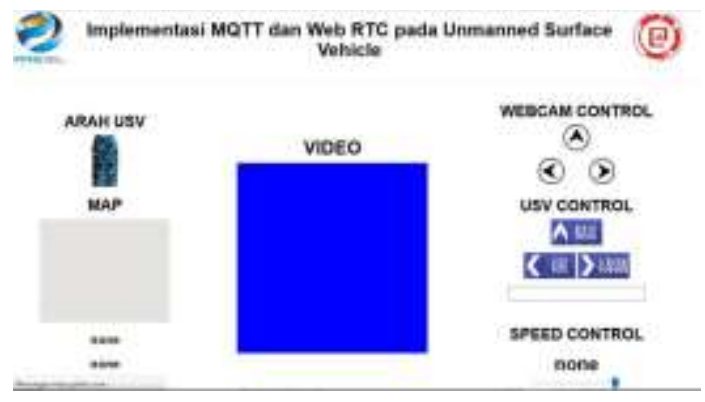

Figure 3. Web Interface Console.

The web interface design consists of a USV rudder direction navigation panel, a navigation direction of webcam panel, a speed control panel, an orientation panel of USV, USV location panel, and a video panel that displays real environmental conditions. This application runs using the MQTT Javascript library called Eclipse Paho. 


\section{Results and Discussion}

For the first test, USV is controlled by web interface without showing the webcam. In Figure 4, it appears when the forward button on the web interface is pressed, the position of the rudder will be straight indicating that USV moves straight.

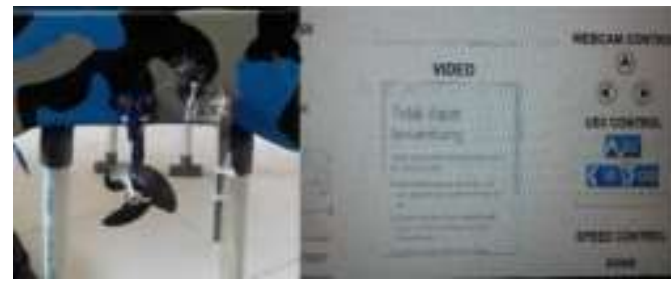

Figure 4. USV is moving Forward.

In Figure 5, it appears when the right button on the web interface is pressed, the position of the rudder will move to the right, indicating that USV turns to the right.

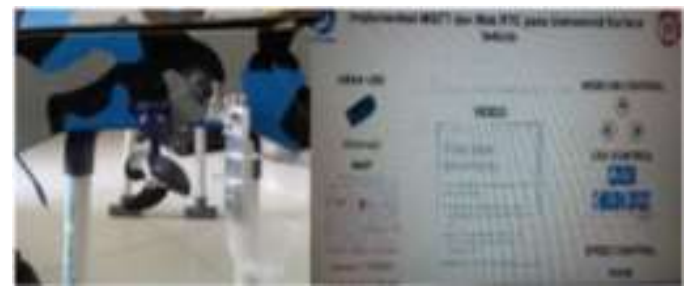

Figure 5. USV is Moving Right Direction.

In Figure 6, it appears when the button for the webcam is pressed to the front to make the webcam position turn towards the front.

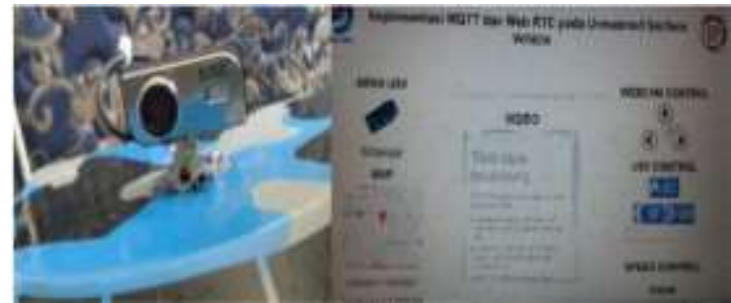

Figure 6. Forwarding direction of a web camera.

In Figure 7, it appears when the button for the webcam is pressed to the right to make the webcam position change towards the right.

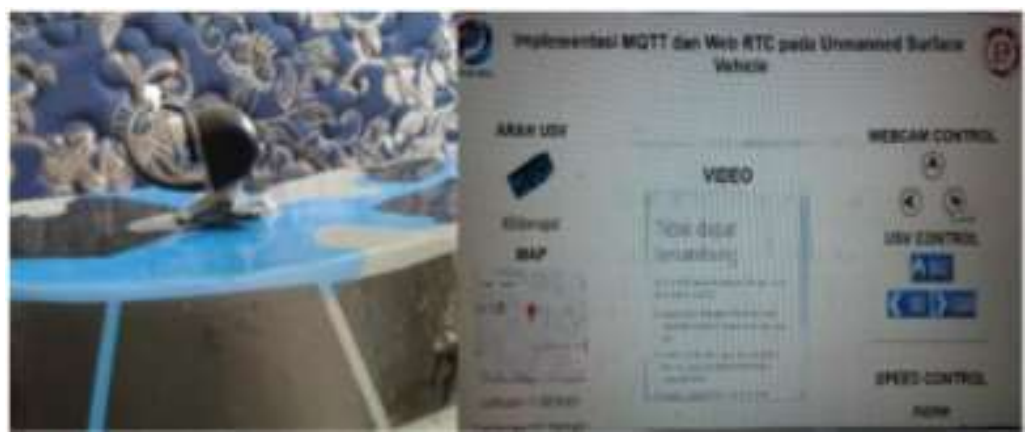

Figure 7. Right Direction of a web camera. 
In Figure 8, it appears when the speed control on the web is set at speed 60, making the USV propeller rotate. This test also shows the compass sensor and GPS running well.

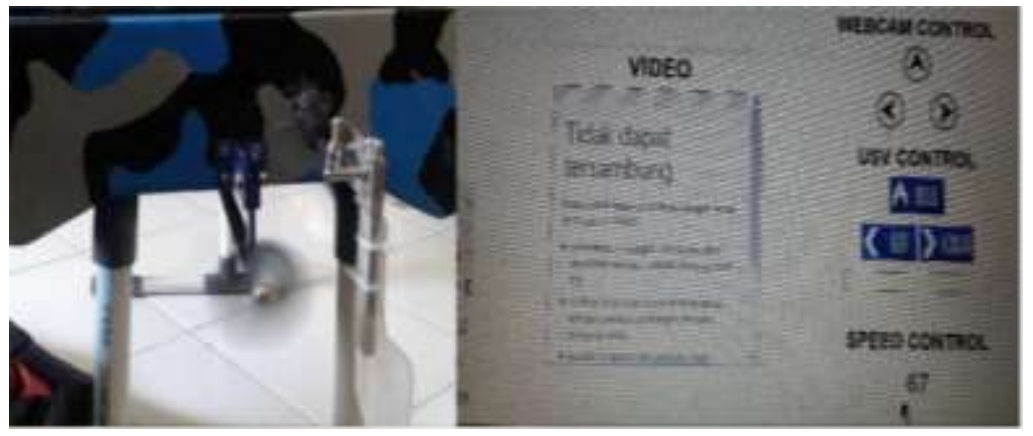

Figure 8. Speed control.

In Figure 9, the web interface displays data from the compass sensor, which is shown by a USV image that can move according to the angle value that appears. Besides that, the web interface also displays the USV position in the map with the latitude and longitude values according to the GPS sensor readings. Data in the compass and GPS section will be updated once every 1 second.

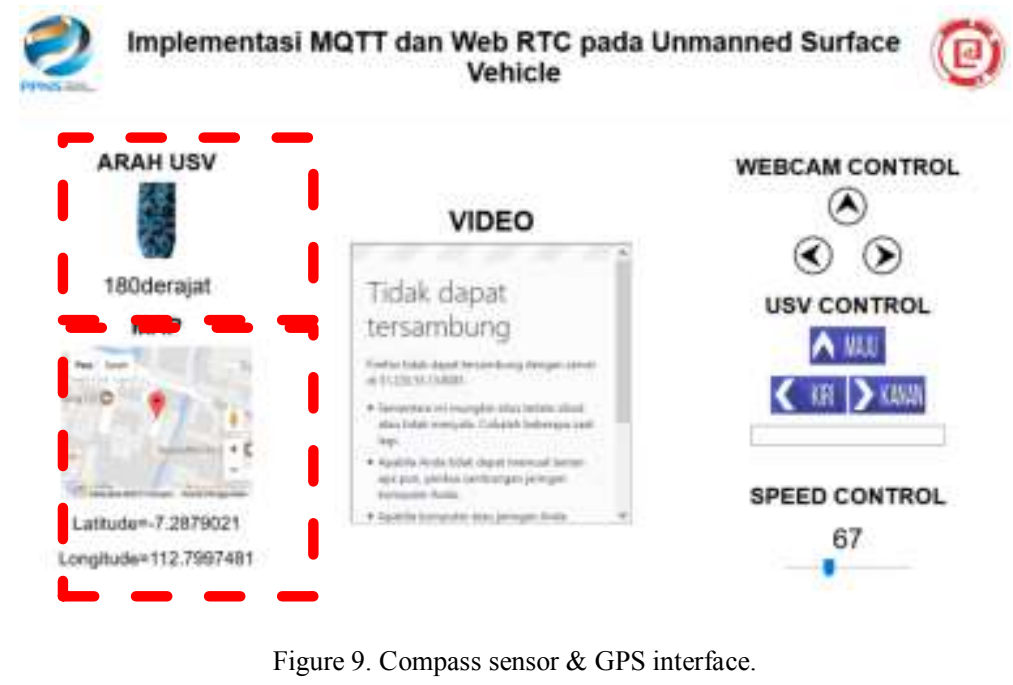

In Figure 10, shows when USV undergoes testing in water. USV can respond to commands given by users through the web interface quite well.

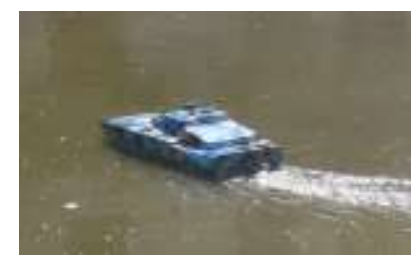

Figure 10. USV Performance on Water.

The following is the USV communication delay time data using the MQTT protocol. In table 2, the command delay sent from the user shows an average of 1.6 seconds.

TABLE 2

AVERAGE DELAY TIME OF USV COMMAND RESPONSE 


\begin{tabular}{lll}
\hline No & Command & Delay time(seconds) \\
\hline 1 & Speed control & 1.92 \\
2 & Webcam direction & 1.44 \\
3 & Rudder direction & 1.44 \\
& Average & 1.6
\end{tabular}

Testing the entire system using the web camera is shown in Figure 11 as follows.

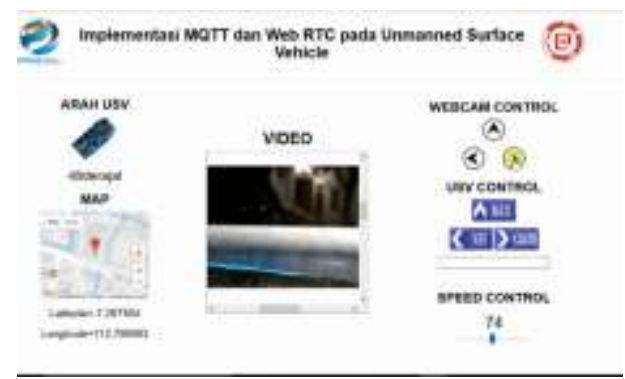

Figure 11. Web interface using MQTT \& WebRTC protocol.

\section{Conclusions and Future Work}

The use of USV to solve problems in the military, security and research sectors is still constrained by USV control capabilities that can only be controlled at close range. This research proposes the development of USV control via an Internet network. The increase in USV's capabilities can benefit the emergence of remote laboratory concept that can be utilised by academics and remote patrol surveillance that is utilised by the military and security sectors. The MQTT protocol as one of the protocols used in the internet of things application was implemented in this project. MQTT is suitable for remote applications controlling and monitoring because it has small data packets that meet the needs of real-time data communication.

This research can be further developed by adding USV facilities that support remote laboratory needs such as the environmental sensors and machine learning algorithm for solving task complexity in water area [21]. Regarding communication, development needs to be done by comparing with other protocols that are developing and used in the Internet of Things application.

Acknowledgments: The work is supported by the Guangxi Science and Technology Major Project (Grant No.AA17204018).

\section{References}

1. J. Veers, V. Bertram, "Development of the USV multi-mission surface vehicle III" In Proceedings of 5th International Conference on Computer Applications and Information Technology in the Maritime Industries, Oegstgeest, pp. 345-355.

2. R.A. Atmoko, A.S. Aisjah, G. Nugroho, "Analysis of Spatial Characteristic of Maritime Weather in Java Sea" In Proceeding of IPTEK Journal of Proceedings Series, 2(1). 2016.

3. J. Jin, J. Zhang, F. Shao, Z. Lyu, D. Wang, "A novel ocean bathymetry technology based on an unmanned surface vehicle" Acta Oceanologica Sinica, 37, 99-106, 2018.

4. D. Kim, H. Kim, S. Jung, J. Koo, J. Kim, J.U Shin, H. Myung, "Development of a jellyfish reconnaissance and removal robot system using unmanned aerial and surface vehicles" In Proceedings of 2015 12th International Conference on Ubiquitous Robots and Ambient Intelligence (URAI), pp. 101-101, 2015. 
5. S. Fahey, Luqi, "Unmanned vehicles for anti-submarine warfare" In Proceedings of OCEANS 2016 MTS/IEEE , pp. 1-4, 2016.

6. T. Yang, S. Hsiung, C. Kuo, Y. Tsai, K. Peng, Y. Hsieh, Z. Shen, J. Feng, C. Kuo, "Development of unmanned surface vehicle for water quality monitoring and measurement" In Proceedings of 2018 IEEE International Conference on Applied System Invention (ICASI), pp. 566-569, 2018.

7. T. Nasution, I. Siregar, M. Yasir, "UAV telemetry communications using ZigBee protocol" In Proceedings of Journal of Physics: Conference Series, p. 012001, 2017.

8. J. Wang, W. Gu, J. Zhu, "Design of an autonomous surface vehicle used for marine environment monitoring“"In Proceedings of 2009 International Conference on Advanced Computer Control, pp. 405-409, 2009.

9. A. Coelho, M. Lopes, B. Ferreira, R. Campos, M. Ricardo, "Experimental evaluation of shore to unmanned surface vehicle Wi-Fi communications" In Proceedings of 2018 Wireless Days (WD), pp. 86-91, 2018.

10. A.L. Christensen, S. Oliveira, O. Postolache, M.J.o. De Oliveira, S. Sargento, P. Santana, L. Nunes, F.J. Velez, P. Sebastiao, V. Costa, "Design of Communication and Control for Swarms of Aquatic Surface Drones" In Proceedings of International Conference on Agents and Artificial Intelligence (ICAART), pp. 548-555.

11. X. Sun, G. Wang, Y. Fan, D. Mu, B. Qiu, "Collision Avoidance of Podded Propulsion Unmanned Surface Vehicle with COLREGs Compliance and Its Modeling and Identification“ IEEE Access, 6, 55473-55491, 2018.

12. M.S Ayas, E. Sahin, İ.H. Altaş, "Performance of PSO based classical and fractional PID controllers for an unmanned surface vehicle“ In Proceedings of 2018 26th Signal Processing and Communications Applications Conference (SIU), pp. 1-4, 2018.

13. Z. Peng, Y. Tian, D. Wang, L. Liu, "Autopilot design for a robotic unmanned surface vehicle" In Proceedings of 2015 34th Chinese Control Conference (CCC), pp. 6116-6120, 2015.

14. C. Viegas, A. Pavani, N. Lima, A. Marques, I. Pozzo, E. Dobboletta, V. Atencia, D. Barreto, F. Calliari, A. Fidalgo, "Impact of a remote lab on teaching practices and student learning" Computers Education, 126, 201-216, 2018.

15. G.R. Alves, A. Fidalgo, A. Marques, C. Viegas, M.C. Felgueiras, R. Costa, N. Lima, M. Castro, G. Díaz-Orueta, E.S.C. Ruiz, "Spreading remote lab usage a system-A community-A Federation" In Proceedings of 2016 2nd International Conference of the Portuguese Society for Engineering Education (CISPEE), pp. 1-7, 2016.

16. J.R. Brinson, "Learning outcome achievement in non-traditional (virtual and remote) versus traditional (hands-on) laboratories: A review of the empirical research", Computers Education, 87, 218-237, 2015.

17. J.E Corter, S.K. Esche, C. Chassapis, J. Ma, J.V Nickerson, "Process and learning outcomes from remotely-operated, simulated, and hands-on student laboratories" Computers Education, 57, 20542067, 2011.

18. R.A. Atmoko, R. Riantini, M.K. Hasin, "IoT real time data acquisition using MQTT protocol" In Proceedings of Journal of Physics: Conference Series, p. 012003, 2016.

19. R.A. Atmoko, D. Yang, "Online Monitoring \& Controlling Industrial Arm Robot Using MQTT Protocol" In Proceedings of 2018 IEEE International Conference on Robotics, Biomimetics, and Intelligent Computational Systems (Robionetics), pp. 12-16, 2018.

20. R.A. Atmoko, Internet of Things with JavaScript (Node.JS + Johnny-five + Socket.IO), Moko Self Publishing, Guilin, 2019.

21. P. O. Hanggara, "Prototype control and monitoring system safety device from leakage ammonia at marine loading arm with comparison of Neural Network (NN) and Extreme Learning Machine (ELM) method", In Proceedings of 2017 International Symposium on Electronics and Smart Devices (ISESD), pp. 85-89, 2017. 